\title{
The Use of a Distal-to-Tactile Sensory Substitution Interface Does Not Lead to Extension of Body Image
}

\author{
Marek McGann ${ }^{*}$ Tom Froese ${ }^{\dagger}$ William Bigge ${ }^{\ddagger}$ Adam Spiers $\wedge$ and Anil K. Seth \\ (*)Mary Immaculate College University of Limerick, Ireland \\ (†) University of Tokyo, Japan \\ (†)Sackler Centre for Consciousness Science, University of Sussex, UK \\ (^)Bristol Robotics Laboratory, University of the West of England, UK \\ E-mail:marek.mcgann@mic.ul.ie,t.froese@gmail.com
}

\begin{abstract}
$A$ range of studies in the past decade and a half indicate significant impacts of tool use on body image. In cases of intentional action, contractions of near space or experienced extensions of limbs have been shown when using tools such as rakes. It remains unclear whether the changes in body image are effected by the tool enabling perception at a distance or action/manipulation of the environment at a distance. We studied this issue using a new research tool, the Enactive Torch, a sensory substitution device specifically designed for research into perception and bodily action. The Enactive Torch allows perception at a distance without the capacity for distal action. We report a first experiment indicating that its use on a navigation task has no effect on body image.
\end{abstract}

\section{Introduction}

In the past fifteen years or so a significant literature has developed around the impact that tool use has on body image and perception. Using tools has shown to have a range of implications for how a person perceives both the space around them and their own bodies.

There remain clear questions as to precisely what the roles of the various aspects of the agent-toolenvironment system are in these changes in bodily perception. In this paper we review some of the research concerning these effects of tools on the user, and report an initial study using an enactive interface, the Enactive Torch (ET, see [1]) which allows a more refined investigation of some of the outstanding issues. A first experiment on the Enactive Torch's impact on body image is reported and some conclusions for our understanding of the relationship between body, tool and environment are outlined.

\section{Tool use, cognition and the body.}

Developments in the neuroscience and psychology of practical tool use have produced evidence for the fluid reconfiguration of body image. Tools do not appear to simply be "peripherals" - appendages to a stable core of cognitive function and bodily awareness. Rather, they are transformative, changing body image and cognition alike [2-4].

Iriki et al. [5] noted measurable effects on the receptive fields of neurons after tool use, suggestive evidence that the tool was incorporated into the body image. Subsequent work from a range of laboratories (see [6] for a review) has reinforced this conclusion.

Work with healthy participants [7] and people with lateral neglect [8] has shown that space and distance are contracted in context of elongated tools (the tools appear to have the effect of bringing the distant closer). More recently Cardinali et al. [9] showed evidence not only for behavioral differences (such that subsequent actions without tools were affected), but also notable changes in actual perceived morphology. After a period of using an elongated "grabber" tool, arm movements without the tool showed longer latencies and reduced maximal amplitude of reaching parameters, consistent with the tool's allowing participants to grasp objects as though their arm was longer than it was. Additionally, when asked, blindfolded, to indicate with their other hand where on their tool-using arm they had felt a touch, their performance indicated that mean distance between perceived distance between their fingertip and elbow

This is an Open Access article distributed under the terms of the Creative Commons Attribution-Noncommercial License 3.0, which permits unrestricted use, distribution, and reproduction in any noncommercial medium, provided the original work is properly cited. 
increased after tool-use. Participants apparently experienced their arms as having become extended.

Understanding the particular relations between the actor and their environment that support these changes in behavior and body image is not easy, though it would seem that the intention to perform some action is crucial. Some researchers [10] have found that changes in perception only occur in cases where the user actively intends to use the tool and others [11] have reported that these changes are only found after active tool use - passive exposure to the tool (holding it but not using it) is not sufficient.

It would seem that the transformed relations between the agent and their distal environment is key to the kinds of effects mentioned above. Identifying the particular aspects of those transformed relations that produce the effects now presents a challenge. Two possibilities are immediately apparent. On the one hand, use of elongated tools such as rakes allow for tactile interaction with the environment at a distance feeling the world at a distance beyond normal reach. On the other hand, such tools also allow for action at a distance - having a direct impact on the environment beyond normal reach by using the tool.

To tease these two possibilities apart, a novel form of tool, that allows for one relation to hold while fixing the other, is required.

\section{The Enactive Torch: A new tool for the study of perception.}

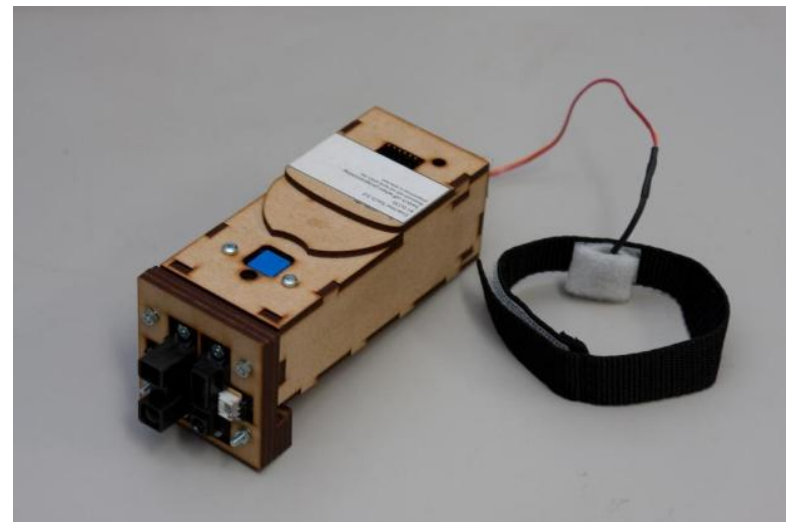

Figure 1. The Enactive Torch. The handheld device has a configurable front-mounted distance sensor. A vibro-tactile actuator is worn on the wrist using the pictured Velcro strap.

The Enactive Torch (ET) (see Figure 1) is an enactive interface specifically designed for the investigation of perception, consciousness and bodily movement.

The ET is a handheld device with a front-mounted sensor. The model used in the study reported here used an infra-red sensor to determine distance to objects, though the sensor is replaceable to allow for a range of possible detectors (e.g. luminescence, temperature, humidity or other environmental variables). Output to the user is via a vibro-tactile motor worn on the hand or wrist.

The particular input-output response profiles of the ET are programmable, though normally the intensity of the vibration is proportional to the current reading from the distance sensor. The device can also transmit (over Bluetooth) and/or store readings from sensor, actuator, or an on-board accelerometer. Recorded actuator data may also be played back through the ET.

\subsection{The Enactive Torch, tool-use and body image}

Configured with an infra-red distance sensor, the Enactive Torch operates in a manner analogous to a blind-person's cane, allowing someone to detect environmental features such as edges and obstacles at a distance of approximately one metre. Unlike a cane, rake or other elongated tool previously used in tool-use research, however, while the Torch allows for perceptual interaction with the environment, it does not offer any possibility to act on the environment at that distance. This makes it an ideal tool for investigating which aspect of tool-use may have an impact on body image.

\section{The impact of navigating with the Enactive Torch on perceived arm length.}

\subsection{Design}

A mixed between-within design was used to assess whether training with and use of the ET produced any changes in body image. Participants in the control group received a five-minute introduction to the use of the device prior to the experimental task - navigating a maze blindfolded using the Torch. Participants in the experimental group received two training sessions (between 1.5 and 2 hours in combined duration) in which a series of tasks, including navigation on a different, simpler maze, were completed. Dependent variables were the number of times the test maze was completed while blindfolded, the number of errors (collisions with the maze edges), and perceived length 
of the arm using the ET both before and after the task. Arm measurements were taken by having the participant hold their arm horizontally beneath a screen, and indicate with a laser pointer onto the screen where they felt a number of touches on the arm at fingertip, wrist and elbow. A standard position on their shoulder was marked at the beginning of testing. The screen was aligned to the mark and all measurements of the arm, perceived and actual, taken from it before and after task performance.

\subsection{Materials}

The Enactive Torch was used by all participants using the same infra-red distance sensor. The device was configured with a standard response profile producing vibro-tactile output inversely proportional to distance from a detected object (that is, the closer to an object the participant approached, the more strongly they experienced the vibration from the Torch).

An irregular maze of roughly rectangular contour and approximately fourteen metres in length was arranged in a classroom from chairs with their backs facing the pathway. The use of waist-high objects limited potential cues available to the participant from differences in general luminance and variations in acoustics of blocked versus open spaces. The beginning and the ending of the maze were positioned on the same side of the room so that participants could easily find their way back to the beginning after completing it.

\subsection{Participants}

Participants were twenty-two undergraduate and postgraduate students of Mary Immaculate College, Limerick. Half were randomly assigned to the training condition. Participation was entirely voluntary.

\subsection{Procedure}

Prior to completing any tasks, measurements were taken of participants' arm length and perceived arm length.

At testing, participants were re-acquainted or briefly introduced to the Enactive Torch and its operation.

Participants were fitted with the ET in their preferred hand and blindfolded. They were then placed at the starting position of the maze (which they had not previously seen). They were told that they would be given five minutes during which they should complete the maze as many times as they could, but in doing so that they should avoid bumping into anything. They were also instructed that each time they would complete the maze they would be told that they had done so, and that they should then try to complete the maze once more from the beginning. In this way the experimenter ensured that participants would not accidentally exit the maze completely during the task.

Bumps were recorded when either the participant's body or the ET came in contact with the maze edges. Each completion of the maze was also recorded. After the defined period of five minutes participants were instructed to stop and remove their blindfold. The procedure for measuring their actual and perceived arm length was then repeated.

\subsection{Results}

Performance on the maze task was generally good. Modal and median number of completions for the combined trained and untrained groups was five. The number of collisions varied considerably, with a range of 9 to 50, and a median of 21. Mann-Whitney U tests were used to determine differences in performance between the trained and untrained groups. No significant difference was found between the trained and untrained groups on the number of completions ( $p$ $=0.58$ ). In order to assess the differences in overall performance between trained and untrained groups a score was calculated for each participant by dividing the number of collisions by the number of completions of the maze. Again, no significant differences were found between trained and untrained groups ( $p=$ $0.715)$.

Measurements of perceived distance between shoulder and elbow, wrist and fingertip provided three scores for each participant both before and after testing..

A between-within MANOVA was used to assess the differences in scores between trained and untrained groups, and before and after the task. There was no statistically significant difference found between trained and untrained on any measurement $(\mathrm{F}(1,20)=$ $0.008 ; p=0.932)$ There was also no significant in perceived arm length before and after the task $(\mathrm{F}(1,20)$ $=2.347 ; p=0.141)$. No interactions reached significance.

These results indicate that there was no significant change in perceived arm length at any of the measured positions after using the Enactive Torch, a device which enables a form of tactile interaction with the environment at a distance without also enabling manipulation of the environment. 


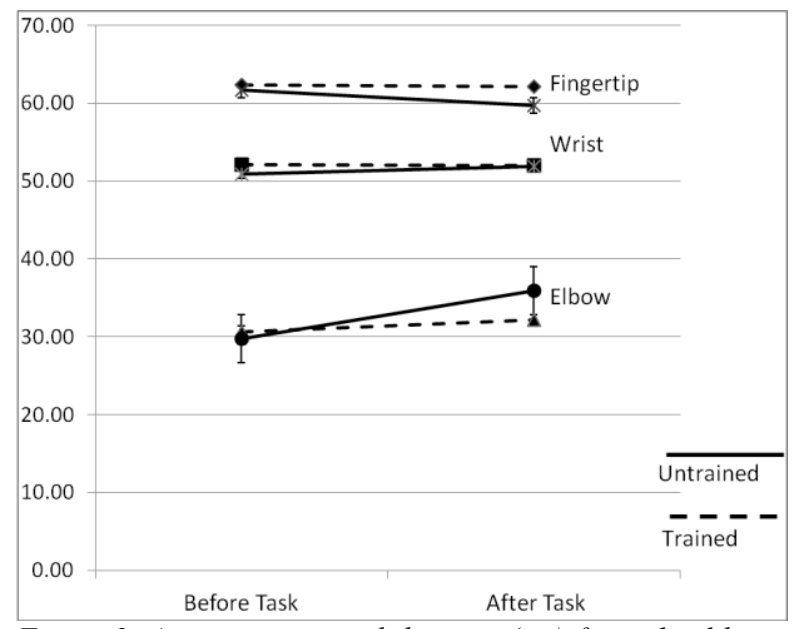

Figure 2. Average perceived distance (cm) from shoulder to elbow, wrist and fingertip before and after navigation task for the trained and untrained groups Error bars are 1 SEM.

\section{Conclusion}

Though it is clear that much work remains to be done, the first results from use of the Enactive Torch are consistent with the hypothesis that it is distal action rather than distal perception that underlies transformation in body image after tool use.

While we recognize the difficulties in inferring conclusions from negative outcomes, our initial finding nonetheless suggests that perceived body image has to do with tracking the relations of potential action between the agent and their environment, rather than being a matter of perception of the body itself per se.

Should these initial findings be borne out through replication they have interesting implications for our understandings of tools, tool-use and cognition. The relationship between perception and action has become a rich domain of research and controversy in recent years. Tools, which provide for a transformation of both, offer a means of examining the perception-action relationship in a dynamic manner. Specifically designed instruments such as the Enactive Torch, by explicitly undermining some of the standard characteristics of handheld tools, while providing an intuitive, embodied mode of perception for users, may prove to be crucial in exploring this issue and divining the nature of the complex of relationships at the heart of embodied cognition and bodily action.

Acknowledgements: This work was supported in part by a donation from the Dr. Mortimer and Theresa Sackler Foundation and by EPSRC grant EP/G007543/1 to AKS. Data collection was supported by an MIC Research Seed Funding Scheme grant to
MMcG. The authors are also very grateful to Alan McAuliffe for his efforts during the experiment.

\section{References}

[1] T. Froese and A. Spiers, "Toward a phenomenological pragmatics of enactive perception," in Proceedings of the 4th International Conference on Enactive Interfaces, Grenoble, France, 2007, pp. 105108.

[2] J. Stewart, "Foundational issues in enaction as a paradigm for cognitive science: From the origin of life to consciousness and writing," in Enaction: Towards a new paradigm of cognitive science, J. Stewart, O. Gapenne, and E. Di Paolo, Eds. Cambridge, Mass: MIT Press, 2010.

[3] E. Hutchins, "Enaction, imagination and insight," in Enaction: Towards a new paradigm of cognitive science, J. Stewart, O. Gapenne, and E. Di Paolo, Eds. Cambridge, Mass: MIT Press, 2010.

[4] V. Havelange, "The ontological constitution of cognition and the epistemological constitution of cognitive science: Phenomenology, enaction and technology," in Enaction: Towards a new paradigm of cognitive science, J. Stewart, O. Gapenne, and E. Di Paolo, Eds. Cambridge, Mass: MIT Press, 2010.

[5] A. Iriki, M. Tanaka, and Y. Iwamura, "Coding of modified body schema during tool use by macaque postcentral neurones.," Neuroreport, vol. 7, no. 14, p. $2325,1996$.

[6] A. Maravita and A. Iriki, "Tools for the body (schema).," Trends in Cognitive Sciences, vol. 8, no. 2, pp. 79-86, Feb. 2004.

[7] M. R. Longo and S. F. Lourenco, "On the nature of near space: Effects of tool use and the transition to far space.," Neuropsychologia, vol. 44, no. 6, pp. 977-981, 2006.

[8] A. Berti and F. Frassinetti, "When far becomes near: Remapping of space by tool use," Journal of Cognitive Neuroscience, vol. 12, no. 3, pp. 415-420, 2000.

[9] L. Cardinali, F. Frassinetti, C. Brozzoli, C. Urquizar, A. C. Roy, and A. Farnè, "Tool-use induces morphological updating of the body schema," Current Biology, vol. 19, no. 12, p. R478-R479, Jun. 2009.

[10] J. K. Witt, D. R. Proffitt, and W. Epstein, “Tool Use Affects Perceived Distance, But Only When You Intend to Use It.," Journal of Experimental Psychology: Human Perception and Performance, vol. 31, no. 5, pp. 880-888, 2005.

[11] A. Farnè, A. Iriki, and E. Làdavas, "Shaping multisensory action-space with tools: Evidence from patients with cross-modal extinction.," Neuropsychologia, vol. 43, no. 2, pp. 238-248, 2005. 